\title{
On Quasi-weak m-power commutative Near - rings and Quasi - weak $(m, n)$ power commutative Near - rings
}

\author{
G.Gopalakrishmoorthy ${ }^{1}$, S.Geetha ${ }^{2}$ and S.Anitha ${ }^{3}$ \\ ${ }^{1}$ Principal, Sri krishnasamy Arts and Science College, Sattur-626203, Tamilnadu. \\ ${ }^{2}$ Dept. of Mathematics, Pannai College of Engineering and Technology, Keelakkandani, Sivagangai - 630561. \\ ${ }^{3}$ Lecturer,Raja Doraisingam Government Arts College, Sivagangai - 630 561, Tamil Nadu.
}

Abstract: $A$ right near - ring $N$ is called weak commutative if $x y z=x z y$ for every $x, y, z \in N$

(Definition 9.4 [10]). A right near - ring $N$ is called pseudo commutative (Definition 2.1 [11] ) if xyz = zyx for all $x, y, z \in N . A$ right near - ring $N$ is called quasi - weak commutative (Definition 2.1 [7] )

if $x y z=y x z$ for all $x, y, z \in N$. We call a right near - ring $N$ to be quasi-weak $m$-power commutative

if $x^{m} y z=y^{m} x z$ for all $x, y, z \in N$. $N$ is said to be Quasi-weak $(m, n)$ power commutative near - ring if

$x^{m} y^{n} z=y^{m} x^{n} z$ for all $x, y, z \in N$.In this paper we study and establish various results of Quasi - weak

$m$ - power commutative near - ring and Quasi - weak $(m, n)$ power commutative near -- ring.

\section{Introduction}

S.Uma,R.Balakrishnan and T.Tamizhchelvam [11] called a near- ring $\mathrm{N}$ to be pseudo commutative if $\mathrm{xyz}=\mathrm{zyx}$ for every $\mathrm{x}, \mathrm{y}, \mathrm{z} \in$ N.G.Gopalakrishnamoorthy and S.Geetha [4] called a ring $\mathrm{R}$ to be $\mathrm{m}$ power commutative if $x^{m} y=y^{m} x$ for all $x, y \in R$ where $m \geq 1$ is a fixed integer.They also called a ring $R$ to be (m,n) power commutative if $x^{m} y^{n}=y^{m} x^{n}$ for all $x, y \in R$ where $m \geq 1$ and $n \geq 1$ are fixed integers. G.Gopalakrishnamoorthy and R.Veega [6] called a near - ring $\mathrm{N}$ to be pseudo m- power commutative if $\mathrm{x}^{\mathrm{m}} \mathrm{y} \mathrm{z}$ $=\mathrm{z} \mathrm{y}^{\mathrm{m}} \mathrm{x}$ for all $\mathrm{x}, \mathrm{y}, \mathrm{z} \in \mathrm{N}$ where $\mathrm{m} \geq 1$ is a fixed integer. G.Gopalakrishnamoorthy, N.Kamaraj and S.Geetha [7] defined a near - ring $N$ to be Quasi - weak commutative if $x y z=y x z$ for all $x, y, z \in N$.

In this paper we define quasi-weak $\mathrm{m}$ - power commutative near - ring and quasi - weak $(\mathrm{m}, \mathrm{n})$ power commutative near - ring and establish some results.

\section{Preliminaries}

Throughout this paper $\mathrm{N}$ denotes a right near - ring with atleast two elements. For any non-empty set $\mathrm{A}$ $\subseteq \mathrm{N}$, we denote $\mathrm{A}-\{0\}$ by $\mathrm{A}^{*}$. In this section we present some known definitions and results which are useful in the development of this paper.

\subsection{Definition [10]}

A near - ring $N$ is called weak-commutative if $x y z=x z y$ for every $x, y, z \in N$.

\subsection{Definition}

A right near- ring $\mathrm{N}$ is said to be distributive near - ring if $\mathrm{a} \cdot(\mathrm{b}+\mathrm{c})=\mathrm{a} \cdot \mathrm{b}+\mathrm{a} \cdot \mathrm{c}$ for all $\mathrm{a}, \mathrm{b}, \mathrm{c} \in \mathrm{N}$.

2.3 Definition [11]

A near - ring $\mathrm{N}$ is called pseudo commutative if $\mathrm{xyz}=\mathrm{zyx}$ for every $\mathrm{x}, \mathrm{y}, \mathrm{z} \in \mathrm{N}$.

2.4 Definition

A near - ring $\mathrm{N}$ is said to be pseudo anti- commutative if $x y z=-$ zyx for every $x, y, z \in N$.

2.5 Definition [6]

A near - ring $\mathrm{N}$ is said to be pseudo $\mathrm{m}$ - power commutative if $\mathrm{x}^{\mathrm{m}} \mathrm{yz}=\mathrm{zy}^{\mathrm{m}} \mathrm{x}$ for all $\mathrm{x}, \mathrm{y}, \mathrm{z} \in \mathrm{N}$.

2.6 Definition [6]

A near - ring $\mathrm{N}$ is said to be pseudo $\mathrm{m}$ - power anti - commutative if $\mathrm{x}^{\mathrm{m}} \mathrm{yz}=-\mathrm{zy}^{\mathrm{m}} \mathrm{x}$ for all $\mathrm{x}, \mathrm{y}, \mathrm{z} \in \mathrm{N}$.

2.7 Lemma [6]

Let $\mathrm{N}$ be a near-ring. If $\mathrm{xyz}= \pm \mathrm{zyx}$ for all $\mathrm{x}, \mathrm{y}, \mathrm{z} \in \mathrm{N}$, then $\mathrm{N}$ is either pseudo Commutative or pseudo anticommutative.

\subsection{Lemma [6]}

Let $\mathrm{N}$ be a near-ring. If $\mathrm{x}^{\mathrm{m}} \mathrm{yz}= \pm \mathrm{z} \mathrm{y}^{\mathrm{m}} \mathrm{x}$ for all $\mathrm{x}, \mathrm{y}, \mathrm{z} \in \mathrm{N}$, then a $\mathrm{N}$ is either pseudo $\mathrm{m}$ - power Commutative or pseudo $\mathrm{m}$ - power anti- commutative.

3.1 Definition[7]

\section{Quasi- weak m- power commutative near - rings}

A near $-\operatorname{ring} \mathrm{N}$ is said to be quasi-weak commutative if $\mathrm{xyz}=\mathrm{yxz}$ for all $\mathrm{x}, \mathrm{y}, \mathrm{z} \in \mathrm{N}$. 3.2 Definition[7] 
A near - ring $\mathrm{N}$ is said to be quasi-weak anti - commutative if $\mathrm{xyz}=-\mathrm{yxz}$ for all $\mathrm{x}, \mathrm{y}, \mathrm{z} \in \mathrm{N}$.

\subsection{Definition}

Let $N$ be a near - ring. $N$ is said to be quasi-weak $m-$ power commutative if $x^{m} y z=y^{m} x z$ for all $x, y, z \in N$, where $m \geq 1$ is a fixed integer.

\subsection{Definition}

Let $\mathrm{N}$ be a near - ring. $\mathrm{N}$ is said to be quasi-weak $\mathrm{m}$ - power anti-commutative if $\mathrm{x}^{\mathrm{m}} \mathrm{yz}=-\mathrm{y}^{\mathrm{m}} \mathrm{x} \mathrm{z}$ for all $\mathrm{x}, \mathrm{y}, \mathrm{z} \in \mathrm{N}$, where $\mathrm{m} \geq 1$ is a fixed integer.

\subsection{Lemma}

Let $\mathrm{N}$ be a distributive near - ring.If $\mathrm{xyz}= \pm \mathrm{yxz}$ for all $\mathrm{x}, \mathrm{y}, \mathrm{z} \in \mathrm{N}$ then $\mathrm{N}$ is either quasi - weak commutative or quasi - weak anti - commutative.

\section{Proof:}

$$
\begin{aligned}
& \text { For each } a \in N \text {, let } \\
& \qquad \begin{aligned}
C_{a} & =\{x \in N / x a z=\operatorname{axz} \forall z \in N\} \\
A_{a} & =\{x \in N / x a z=-\operatorname{axz} \forall z \in N\}
\end{aligned}
\end{aligned}
$$

By the hypothesis of the lemma,

$$
\mathrm{N}=\mathrm{C}_{\mathrm{a}} \cup \mathrm{A}_{\mathrm{a}}
$$

We note that if $\mathrm{x}, \mathrm{y} \in \mathrm{C}_{\mathrm{a}}$, then $\mathrm{x}-\mathrm{y} \in \mathrm{C}_{\mathrm{a}}$.

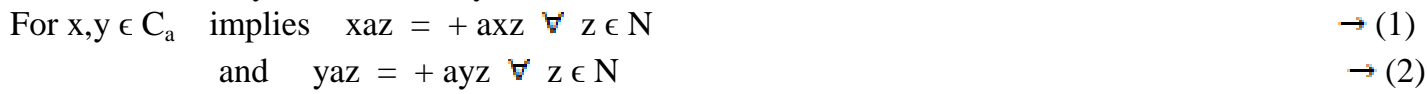

$$
\begin{aligned}
& \text { (1) }- \text { (2) gives } \\
& (\mathrm{x}-\mathrm{y}) \mathrm{az}=\mathrm{a}(\mathrm{x}-\mathrm{y}) \mathrm{z} \forall \mathrm{z} \in \mathrm{N}
\end{aligned}
$$

which implies $(x-y) \in C_{a}$.

Similarly, if $x, y \in A_{a}$, then $x-y \in A_{a}$

We claim that either $\mathrm{N}=\mathrm{C}_{\mathrm{a}}$ or $\mathrm{N}=\mathrm{A}_{\mathrm{a}}$.

Suppose $N \neq C_{a}$ and $N \neq A_{a}$, then there are elements $b \in C_{a}-A_{a}$ and

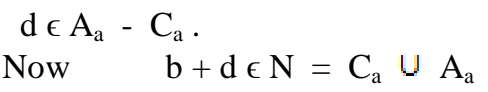

If $b+d \in C_{a}$ then $d=(b+d)-b \in C_{a}$, a contradiction.

If $\mathrm{b}+\mathrm{d} \epsilon \mathrm{A}_{\mathrm{a}}$ then $\mathrm{b}=(\mathrm{b}+\mathrm{d})-\mathrm{d} \epsilon \mathrm{A}_{\mathrm{a}}$, again a contradiction.

Hence either $\mathrm{N}=\mathrm{C}_{\mathrm{a}}$ or $\mathrm{N}=\mathrm{A}_{\mathrm{a}}$

Let $\quad \mathrm{A}=\left\{\mathrm{a} \in \mathrm{N} / \mathrm{C}_{\mathrm{a}}=\mathrm{N}\right\}$

and $\quad B=\left\{a \in N / A_{a}=N\right\}$

Clearly $\mathrm{N}=\mathrm{A} \cup \mathrm{B}$.

We note that that if $x, y \in A$, then $x-y \in A$.

For if $\mathrm{x}, \mathrm{y} \in \mathrm{A} \Rightarrow \mathrm{C}_{\mathrm{x}}=\mathrm{N}$ and $\mathrm{C}_{\mathrm{y}}=\mathrm{N}$.

This implies axz $=x a z$ and ayz $=$ yaz for all $a, z \in N$,

So $a(x-y) z=(x-y)$ az for all $a, z \in N$, which proves that $x-y \in A$.

Similarly, if $\mathrm{x}, \mathrm{y} \in \mathrm{B}$, then $\mathrm{x}-\mathrm{y} \in \mathrm{B}$.

We claim that either $\mathrm{N}=\mathrm{A}$ or $\mathrm{N}=\mathrm{B}$.

Suppose $N \neq A$ and $N \neq B$,there are elements $u \in A-B$ and $v \in B-A$.

Now, $\quad u+v \in N=A \cup B$.

If $u+v \in A$, then $v=(u+v)-u \in A$, a contradiction.

If $u+v \in B$, then $u=(u+v)-v \in B$, again a contradiction.

Hence either $\mathrm{N}=\mathrm{A}$ or $\mathrm{N}=\mathrm{B}$.

This proves that $\mathrm{N}$ is either quasi - weak commutative or quasi - weak anti - commutative.

\subsection{Lemma :}

Let $\mathrm{N}$ be a near - ring ( not necessarily associative ) satisfying $(\mathrm{x}-\mathrm{y}){ }^{\mathrm{m}}=\mathrm{x}^{\mathrm{m}}-\mathrm{y}^{\mathrm{m}}$ for all $\mathrm{x}, \mathrm{y} \in \mathrm{N}$, where $\mathrm{m}$ $\geq 1$ is a fixed integer.If $x^{m} y z= \pm y^{m} x z$ for all $x, y, z \in N$, then $N$ is either quasi - weak $m$ - power commutative or quasi - weak $\mathrm{m}$ - power anti-commutative.

\section{Proof:}

For each $a \in N$, let

$$
\begin{aligned}
& C_{a}=\left\{x \in N / x^{m} a z=a^{m} x z \forall\right. \\
& A_{a}=\left\{x \in N / x^{m} a z=-a^{m} x z \forall z \in N\right\}
\end{aligned}
$$


By the hypothesis of the lemma,

$$
\mathrm{N}=\mathrm{C}_{\mathrm{a}} \cup \mathrm{A}_{\mathrm{a}}
$$

We note that, if $x, y \in C_{a}$ then $x-y \in C_{a}$

For $\mathrm{x}, \mathrm{y} \in \mathrm{C}_{\mathrm{a}}$ implies $\mathrm{x}^{\mathrm{m}} \mathrm{a} \mathrm{z}=\mathrm{a}^{\mathrm{m}} \mathrm{xz} \forall \forall \mathrm{z} \in \mathrm{N} \quad \rightarrow$ (1)

$$
\text { and } y^{\mathrm{m}} \mathrm{az}=\mathrm{a}^{\mathrm{m}} \mathrm{yz} \forall \mathrm{z} \in \mathrm{N} \quad \rightarrow \text { (2) }
$$

Equation (1) - (2) gives,

$$
\begin{aligned}
& \left(x^{m}-y^{m}\right) a z=a^{m}(x-y) z \quad \forall z \in N . \\
& \quad \Rightarrow(x-y){ }^{m} a z=a^{m}(x-y) z \forall z \in N . \\
& \Rightarrow(x-y) \in C_{a} .
\end{aligned}
$$

Similarly $x, y \in A_{a}$ implies $x-y \in A_{a}$.

We claim that either $\mathrm{N}=\mathrm{C}_{\mathrm{a}}$ or $\mathrm{N}=\mathrm{A}_{\mathrm{a}}$.

Suppose $N \neq C_{a}$ and $N \neq A_{a}$, there are elements $b \in C_{a}-A_{a}$ and $d \in A_{a}-C_{a}$.

Now, $b+d \in N=C_{a} \cup A_{a}$.

If $b+d \in C_{a}$ then $d=(b+d)-b \in C_{a}$, a contradiction.

Similarly, if $b+d \in A_{a}$, then $b=(b+d)-d \in A_{a}$, again a contradiction.

Hence either $\mathrm{N}=\mathrm{C}_{\mathrm{a}}$ or $\mathrm{N}=\mathrm{A}_{\mathrm{a}}$.

Let $\quad A=\left\{a \in N / C_{a}=N\right\}$

and $B=\left\{a \in N / A_{a}=N\right\}$

Clearly $\mathrm{N}=\mathrm{A} \cup \mathrm{B}$.

We note that if $\mathrm{x}, \mathrm{y} \in \mathrm{A}$ implies $\mathrm{x}-\mathrm{y} \in \mathrm{A}$.

For if $\mathrm{x}, \mathrm{y} \in \mathrm{A}$ implies $\mathrm{C}_{\mathrm{x}}=\mathrm{N}$ and $\mathrm{C}_{\mathrm{y}}=\mathrm{N}$.

This implies $a^{m} x z=x^{m} a z$ and $a^{m} y z=y^{m} a z$ for all $a, z \in N$.

So, $a^{m}(x-y) z=\left(x^{m}-y^{m}\right)$ a $z$ for all $a, z \in N$,

(i.e.,) $a^{m}(x-y) z=(x-y)^{m}$ a $z$ for all $a, z \in N$, which proves that $x-y \in A$.

Similarly $\mathrm{x}, \mathrm{y} \in \mathrm{B}$ implies $\mathrm{x}-\mathrm{y} \in \mathrm{B}$.

We claim that either $\mathrm{N}=\mathrm{A}$ or $\mathrm{N}=\mathrm{B}$.

Suppose $N \neq A$ and $N \neq B$, there are elements $u \in A-B$ and $v \in B-A$.

Now, $\mathrm{u}+\mathrm{v} \in \mathrm{N}=\mathrm{A} \cup \mathrm{B}$.

If $u+v \in A$, then $v=(u+v)-u \in A$, a contradiction.

If $u+v \in B$, then $u=(u+v)-v \in B$, again a contradiction.

Hence either $\mathrm{N}=\mathrm{A}$ or $\mathrm{N}=\mathrm{B}$.

This proves that $\mathrm{N}$ is either quasi-weak $\mathrm{m}$ - power commutative or quasi- weak $\mathrm{m}$ - power anti - commutative.

\subsection{Note :}

When $\mathrm{m}=1$,we get Lemma 3.5 .

3.8 Definition :

Let $N$ be a near-ring and $m \geq 1$ and $n \geq 1$ be fixed integers. $N$ is said to be quasi- weak $(m, n)$

Power commutative, if $x^{m} y^{n} z=y^{m} x^{n} z$ for all $x, y, z \in N$.

3.9 Definition:

Let $N$ be a near-ring and $m \geq 1$ and $n \geq 1$ be fixed integers. $N$ is said to be quasi-weak (m,n) Power anti - commutative, if $x^{m} y^{n} z=-y^{m} x^{n} z$ for all $x, y, z \in N$.

\subsection{Lemma:}

Let $\mathrm{N}$ be a near - ring ( not necessarily associative ) satisfying $(\mathrm{x}-\mathrm{y})^{\mathrm{k}}=\mathrm{x}^{\mathrm{k}}-\mathrm{y}^{\mathrm{k}}$ for $\mathrm{k}=\mathrm{m}, \mathrm{n}$ where $\mathrm{m} \geq 1$ and $n \geq 1$ are fixed integers. If $x^{m} y^{n} z= \pm y^{m} x^{n} z$ for all $x, y, z \in N$, then $N$ is either quasi- weak (m.n)- power Commutative or quasi- weak $(m, n)$ - power anti-commutative.

\section{Proof:}

For each a $€ \mathrm{~N}$, let

$$
\begin{aligned}
& C_{a}=\left\{x \in N / x^{m} a^{n} z=a^{m} x^{n} z \forall z \in N\right\} \\
& A_{a}=\left\{x \in N / x^{m} a^{n} z=-a^{m} x^{n} z \forall z \in N\right\}
\end{aligned}
$$

By the hypothesis of the lemma,

$$
\mathrm{N}=\mathrm{C}_{\mathrm{a}} \cup \mathrm{A}_{\mathrm{a}}
$$

We note that, if $x, y \in C_{a}$ then $x-y \in C_{a}$

For $\mathrm{x}, \mathrm{y} \in \mathrm{C}_{\mathrm{a}}$ implies $\mathrm{x}^{\mathrm{m}} \mathrm{a}^{\mathrm{n}} \mathrm{z}=\mathrm{a}^{\mathrm{m}} \mathrm{x}^{\mathrm{n}} \mathrm{z} \forall \mathrm{z} \in \mathrm{N} \quad \rightarrow$ (1) 


$$
\text { and } \mathrm{y}^{\mathrm{m}} \mathrm{a}^{\mathrm{n}} \mathrm{z}=\mathrm{a}^{\mathrm{m}} \mathrm{y}^{\mathrm{n}} \mathrm{z} \forall \mathrm{z} \in \mathrm{N} \quad \rightarrow(2)
$$

Equation (1) - (2) gives,

$$
\begin{aligned}
& \left(x^{m}-y^{m}\right) a^{n} z=a^{m}\left(x^{n}-y^{n}\right) z \forall z \in N . \\
& \quad \Rightarrow(x-y){ }^{m} a^{n} z=a^{m}(x-y)^{n} z \forall z \in N . \\
& \Rightarrow(x-y) \in C_{a} .
\end{aligned}
$$

Similarly $x, y \in A_{a}$ implies $x-y \in A_{a}$.

We claim that either $\mathrm{N}=\mathrm{C}_{\mathrm{a}}$ or $\mathrm{N}=\mathrm{A}_{\mathrm{a}}$.

Suppose $N \neq C_{a}$ and $N \neq A_{a}$, there are elements $b \in C_{a}-A_{a}$ and $d \in A_{a}-C_{a}$.

Now, $\quad b+d \in N=C_{a} \cup A_{a}$.

If $b+d \in C_{a}$ then $d=(b+d)-b \in C_{a}$, a contradiction.

Similarly, if $b+d \in A_{a}$, then $b=(b+d)-d \in A_{a}$, again a contradiction.

Hence either $\mathrm{N}=\mathrm{C}_{\mathrm{a}}$ or $\mathrm{N}=\mathrm{A}_{\mathrm{a}}$.

Let $\quad A=\left\{a \in N / C_{a}=N\right\}$

and $B=\left\{a \in N / A_{a}=N\right\}$

Clearly $\mathrm{N}=\mathrm{A} \cup \mathrm{B}$.

We note that if $\mathrm{x}, \mathrm{y} \in \mathrm{A}$ implies $\mathrm{x}-\mathrm{y} \in \mathrm{A}$.

For if $x, y \in A$ implies $C_{x}=N$ and $C_{y}=N$.

This implies $a^{m} x^{n} z=x^{m} a^{n} z$ and $a^{m} y^{n} z=y^{m} a^{n} z$ for all $a, z \in N$. $\Rightarrow a^{m}\left(x^{n}-y^{n}\right) z=\left(x^{m}-y^{m}\right) a^{n} z$ for all $a, z \in N$.

So, $a^{m}(x-y)^{n} z=(x-y)^{m} a^{n} z$ for all $a, z \in N$, which proves that $x-y \in A$.

Similarly $x, y \in B$ implies $x-y \in B$.

We claim that either $\mathrm{N}=\mathrm{A}$ or $\mathrm{N}=\mathrm{B}$.

Suppose $N \neq A$ and $N \neq B$, there are elements $u \in A-B$ and $v \in B-A$.

Now, $\mathrm{u}+\mathrm{v} \in \mathrm{N}=\mathrm{A} \cup \mathrm{B}$.

If $u+v \in A$, then $v=(u+v)-u \in A$, a contradiction.

If $u+v \in B$, then $u=(u+v)-v \in B$, again a contradiction.

Hence either $\mathrm{N}=\mathrm{A}$ or $\mathrm{N}=\mathrm{B}$.

This proves that $\mathrm{N}$ is either quasi-weak $(\mathrm{m}, \mathrm{n})$ - power commutative or quasi-weak $(\mathrm{m}, \mathrm{n})$ - power anti-commutative.

\subsection{Note:}

When $\mathrm{m}=\mathrm{n}=1$, we get Lemma 3.5.

When $\mathrm{n}=1$, we get Lemma 3.6.

\section{References}

[1]. H.E.Bell,Quasi Centres, Quasi Commutators, and Ring Commutativity,Acta Maths,Hungary 4( 1 - 2 )(1983), $127-136$.

[2]. L.O.Chung and Jiang Luh,Scalar Central elements in an algebra over a Principal ideal domain,Acta Sci. Maths 41,(1979),289-293

[3]. .G.Gopalakrishnamoorthy and R.Veega, On Quasi - Periodic,Generalised Quasi-Periodic Algebras,Jour.of Inst. of Mathematics and Computer Sciences, Vol 23,No 2 (2010).

[4]. G.Gopalakrishnamoorthy and S.Geetha,On $(\mathrm{m}, \mathrm{n})$ - Power Commutativity of rings and Scalar $(\mathrm{m}, \mathrm{n})$ - Power Commutativity of Algebras,Jour. of Mathermatical Sciences ,Vol 24(3),2013, 97-110.

[5]. G.Gopalakrishnamoorthy and R.Veega,On Scalar Power Central Elements in an Algrbra over a Principal ideal domain,Jour. of Mathematical Sciences,Vol 24(3),2013,111-128.

[6]. G.Gopalakrishnamoorthy and R.Veega,On pseudo $\mathrm{m}$ - Power Commutative near - rings and Pseudo (m,n) PowerCommutative near - rings,International Journal of Mathematical Research and Science,Vol (4), 2013,71 - 80 .

[7]. G.Gopalakrishnamoorthy,Kamaraj and S.Geetha,On quasi - weak Commutative Near - rings, International Journal of Mathamatics Research,Vol 5(5),2013,431- 440.

[8]. G.Gopalakrishnamoorthy,S.Geetha and S.Anitha, On Quasi - weak Commutative Boolean - like Near - Rings,Malaya Journal of Matematik,accepted.

[9]. G.Gopalakrishnamoorthy,S.Geetha and S.Anitha, On Quasi Weak Commutative Near - Rings II, Malaya Journal of Matematik ,accepted.

[10]. PilzGiinter,Near - rings,North Holland,Amerterdam,1983.

[11]. S.Uma,R.Balakrishnan and T.Tamizh Chelvam,Pseudo Commutative Near- rings,Scientia Magna, Vol (2010),No 2,75-85. 
